UMD-PP-00-066

\title{
Mirror Dark Matter
}

\author{
R. N. Mohapatra ${ }^{1}$ and V. L. Teplitz ${ }^{2,3}$ \\ ${ }^{1}$ Department of Physics, University of Maryland, College Park, MD, 20742 \\ 2 Office of Science and Technology, Old Executive Office Building, Washington DC, 20502 \\ 3 Department of Physics, Southern Methodist University, Dallas, TX-75275 (permanent address).
}

(March, 2000)

\begin{abstract}
There appear to be three challenges that any theory of dark matter must face: (i) why is $\Omega_{D M}$ of the same order as $\Omega_{\text {Baryons }}$ ? (ii) what are the near solar mass objects $\left(\sim 0.5 M_{\odot}\right)$ observed by the MACHO microlensing project ? and (iii) understanding the shallow core density profile of the halos of dwarf as well as low surface brightness galaxies. The popular cold dark matter candidates, the SUSY LSP and the axion fail to meet these challenges. We argue that in the mirror model suggested recently to explain the neutrino anomalies, the mirror baryons being 15-20 times heavier than familiar baryons, can play the role of the cold dark matter and provide reasonable explanation of all three above properties without extra assumptions.
\end{abstract}

\section{INTRODUCTION}

Dark matter constitutes the bulk of the matter in the universe and a proper understanding of the nature of the new particle that plays this role has profound implications not only for cosmology but also for particle physics beyond the standard model [1]. It is therefore not surprising that one of the major areas of research in both particle physics and cosmology continues to be the physics of dark matter.

Apart from the simple requirement that the right particle physics candidate must have properties that allow it to have the requisite relic density and mass to dominate the mass content of the universe, it should be required to provide a satisfactory resolution of three puzzles of dark matter physics: (i) why is it that the contribution of baryons to the mass density $(\Omega)$ of the universe is almost of the same order as the contribution of the dark matter to it ? (ii) how does one understand the dark objects with mass $\sim 0.5 M_{\odot}$ observed in the MACHO experiment [2], which are supposed to constitute up to $20 \%$ of the mass [3] of the halo of the Milky way galaxy and presumably be connected to the dark constituent that contributes to $\Omega$ ? and, finally (iii) what explains the density profile of dark matter in galactic halos; in particular, the evidence that the core densities of galactic halos remain constant as the radius goes to zero. 
There are many particle physics candidates for dark constituent of the universe. Generally speaking, the prime consideration that leads to such candidates is that they yield the right order of magnitude for the relic density and mass necessary to get the desired $\Omega_{D M} \approx 0.2-1$. This is, of course, the minimal criterion. It requires that the annihilation cross section of the particles must be in a very specific range correlated with their mass. The most widely discussed candidates are the lightest supersymmetric particle (LSP) and the Peccei-Quinn particle, the axion. The first one is expected to have a mass in the range of $100 \mathrm{GeV}$ whereas the mass of the second would be in the range of $\sim 10^{-6} \mathrm{eV}$. The present consensus seems to be that value of $\Omega_{C D M}$ is around $0.2-0.3$, with $\Omega_{\Lambda}$ making up the rest of the energy density of the universe at the moment. Compare these values with $\Omega_{B} \simeq 0.05$. The CDM contribution and the baryon contribution to $\Omega$ are roughly of same order. On the other hand, the nucleon mass is very different from the masses of either the axion or the SUSY LSP. So to understand within the SUSY or the axion models why $\Omega_{B} \sim \Omega_{D M}$, one needs to work in a special range for the parameters of the theory. In either of these pictures, the MACHO observations must have a separate explanation.

Furthermore, in recent years it has been emphasized that the LSP and the axion may also have difficulty in explaining the observed core density behaviour of dwarf speroidal galaxies which are known to be dark matter dominated. The point here is that both the axions and neutralinos, being collisionless and nonrelativistic, accumulate at the core of galactic halos, leading to a core density $\rho(R)$ which goes like $R^{-2}$ rather than a constant which seems to fit data better [4]. We will refer to this as the core density puzzle [8 10].

Spergel and Steinhardt [8] have recently revived an old idea [11], that dark matter may be strongly self interacting, to resolve this puzzle. They argue that, for the right range of the parameters of the particle, it may lead to a halo core which is much less dense and hence in better agreement with observations. Specifically they note that if the dark matter particle has mean free collision path of about a kpc to a Mpc, then the core on this scale cannot "keep on accumulating" dark matter particles, since these will now scatter and "diffuse out". For typical dark matter particle densities of order of one particle per $\mathrm{cm}^{3}$, this requires a cross section for scattering of $\sigma \simeq 10^{-21}-10^{-24} \mathrm{~cm}^{2}$. Furthermore the properties of the dark matter particle must be such that it must not allow for dissipation of the thermal energy via emission of light particles; otherwise, the galactic core would cool and lead to an increase in core density. If these considerations stand the test of time, a theoretical challenge would be to look for alternative dark matter candidates (different from the popular ones described above) and the associated scenarios for physics beyond the standard model.

A class of models known as mirror universe models have recently been discussed. These are motivated theoretically by string theory and experimentally by neutrino physics. They predict the existence of a mirror sector of the universe with matter and force content identical to the familiar sector (prior to symmetry breaking) [12 15. Symmetry breaking might either keep the mirror symmetry exact or break it. This leads to two classes of mirror models: the symmetric mirror model, where all masses and forces in the two sectors remain the same after symmetry breaking [14] and the asymmetric mirror model [13] where the masses in the mirror sector are larger than those in the familiar sector. The mirror particles interact with the mirror photon and not the familiar photon so that they remain dark to our observations. Since the the lightest particles of the mirror sector (other than the neutrinos), the mirror proton and the mirror electron (like those in the familiar sector) are stable and will have 
abundances similar to the familiar protons and electrons, the proton being heavier could certainly qualify as a dark matter candidate. We will show in the next section they can indeed play this role.

It has been shown [16, 17 that, consistent with the cosmological constraints of the mirror universe theory, the mirror baryons have the desired relic density to play the role of dark matter of the universe. The additional neutrinos of the mirror sector are the sterile neutrinos that appear to be needed in order to have a simultaneous understanding of the three different observed neutrino oscillations i.e. solar, atmospheric and the LSND observations. In fact, one view of neutrino oscillation explanations of these phenomena fixes the ratio of familiar particle mass to mirror particle mass thereby narrowing down the freedom in mirror sector parameters. If indeed sterile neutrinos turn out to be required, mirror universe theory is one of the few models where they appear naturally with masses in the desired range. If we denote the mass ratio $m_{p^{\prime}} / m_{p}=\zeta$, then a value of $\zeta \sim 10-30$ is required to explain the neutrino puzzles. What is more interesting is that for the same parameter range required to solve the neutrino puzzles, mirror matter can also provide an explanation of the microlensing observations [16]- in particular why the observed MACHOs have a mass very near the solar mass and are still dark.

\section{ASYMMETRIC MIRROR MODEL IN BRIEF}

Let us start with a brief overview of the mirror matter models [13,14. The basic idea of the model is extremely simple: duplicate the standard model or any extension of it in the gauge symmetric Lagrangian and allow for the possibility that symmetry breaking may be different in the two sectors. (See table below) There is an exact mirror symmetry connecting the Lagrangians (prior to symmetry breaking) describing physics in each sector. Clearly the $W^{\prime} s, \gamma^{\prime} s$ etc in each sector differ from those in the other as do the quarks and leptons. When the symmetry breaking scale is different in the two sectors, we will call this the asymmetric mirror model [13]. The QCD scale being an independent scale in the theory could be arbitrary. We will allow both the weak scale and the QCD scale of the mirror sector to differ from those of the familiar sector [16] and assume the same common ratio $\zeta$ for both scales i.e. $<H^{\prime}>/<H>=\Lambda^{\prime} / \Lambda \equiv \zeta$.

$$
\begin{array}{ccc}
u, d, e, \nu_{e} & \leftrightarrow & u^{\prime}, d^{\prime}, e^{\prime}, \nu_{e}^{\prime} \\
W, Z, \gamma, G & \leftrightarrow & W^{\prime}, Z^{\prime}, \gamma^{\prime}, G^{\prime} \\
\phi, \nu_{R}, \ldots & \leftrightarrow & \phi^{\prime}, \nu_{R}^{\prime}, \ldots
\end{array}
$$

\section{$\leftarrow$ Gravity $\rightarrow$}

It is assumed that the two sectors in the universe are connected by only gravitational interactions. It was shown in [13,14] that gravity induces nonrenormalizable operators that generate mixings between the familiar and the mirror neutrinos. This is one of the ingredients in the resolution of neutrino puzzles. To get an idea of how this works, note that the lepton 
operators induced due to nonperturbative gravitational effects have the form $\frac{L H L H}{M_{P l}}, \frac{L H L^{\prime} H^{\prime}}{M_{P l}}$ and $\frac{L^{\prime} H^{\prime} L^{\prime} H^{\prime}}{M_{P l}}$. After spontaneous breakdown (i.e. $\langle H\rangle=v$ and $\left\langle H^{\prime}\right\rangle=v^{\prime}$, we get for the mass matrix mixing the first generation neutrinos from each sector to have the form (in the basis $\left.\nu_{e}, \nu_{e}^{\prime}\right)$ :

$$
M=\frac{v^{2}}{M_{P l}}\left(\begin{array}{cc}
1 & \zeta \\
\zeta & \lambda \zeta^{2}
\end{array}\right)
$$

where $\zeta \equiv v^{\prime} / v$ defined above. To solve the solar neutrino puzzle via small angle MSW solution, we will choose $\lambda \sim 1$ and $\zeta \approx 10-30$. This gives the sterile neutrino mass of order $10^{-3} \mathrm{eV}$ or so, choosing $M_{P l} \simeq 10^{18} \mathrm{GeV}$, which is also in the right range to explain the solar neutrino puzzle. Note that these are meant to indicate that the model leads to numbers in the right ball park. Emboldened by this result, we will consider the asymmetric version and look at its cosmological implications.

In discussing cosmology, we first note that both sectors of the universe will evolve according to the rules of the usual big bang model except that the cosmic soups in the two sectors may have different temperature. In fact the constraints of big bang nucleosynthesis require that the post inflation reheat temperature in the mirror sector $T_{R}^{\prime}$ be slightly lower than that in the familiar sector $T_{R}$ (define $\beta \equiv T_{R}^{\prime} / T_{R}$ ) so that the contribution of the light mirror particles such as $\nu^{\prime}, \gamma^{\prime}$ etc. to nucleosynthesis is not too important. This is called asymmetric inflation and can be implemented in different ways 18 . We will see that, if we want the mirror nucleons to play the role of the dark matter, we will need a definite value of $\beta$ depening on the choice of $\zeta$. This in turn will help us to predict a value for the equivalent extra neutrinos at the BBN epoch (i.e. $\delta N_{\nu}$ ).

Before detailed discussion, let us first note the impact of the asymmetry on physical parameters and processes. First it implies that $m_{i} \rightarrow \zeta m_{i}$ with $i=n, p, e, W, Z$. This has important implications for the nuclear and atomic physics of the mirror sector [13,23]. For instance, the binding energy of mirror hydrogen is $\zeta$ times larger so that the recombination in the mirror sector takes place much earlier than in the visible sector. With $\beta \equiv T_{R}^{\prime} / T_{R}$ as above, the mirror recombination occurs when the temperature of the familiar sector is $\zeta / \beta T_{r}$ where $T_{r}$ is the recombination temperature in the familiar sector. The mirror sector recombination takes place before familiar sector recombination; this means that density inhomogeneities in the mirror sector begin to grow earlier and familiar matter can fall into them later as in typical cold dark matter scenarios.

\section{MIRROR NUCLEON AS DARK MATTER}

One can also compute the contribution of mirror baryons to the mass density of the universe as follows:

$$
\frac{\Omega_{B^{\prime}}}{\Omega_{B}} \simeq \beta^{3} \zeta
$$

Here we have assumed that baryon to photon ratio in the familiar and the mirror sectors are the same as would be expected since the dynamics are same in both sectors due to mirror symmetry. Eq. (2) implies that both the baryonic and the mirror baryon contribution to $\Omega$ 
are roughly of the same order, as observed. This provides a resolution of the first conceptual puzzle. Furthermore if we take $\Omega_{B} \simeq 0.05$, then $\Omega_{B^{\prime}} \simeq 0.2$ would require that $\beta=(4 / \zeta)^{1 / 3}$. From this one can calculate the effective $\delta N_{\nu}$ using the following formula:

$$
\delta N_{\nu}=3 \beta^{4}+\frac{4}{7} \beta^{4}\left(\frac{11}{4}\right)^{4 / 3}
$$

where the last factor $(11 / 4)^{4 / 3}$ is due to the reheating of the mirror photon gas subsequent to mirror $e^{+^{\prime}} e^{-^{\prime}}$ annihilation. For $\zeta=20$, this implies $\delta N_{\nu} \simeq 0.6$ and it scales with $\zeta$ as $\zeta^{-4 / 3}$. Thus in principle the idea that mirror baryons are dark matter could be tested by more accurate measurements of primordial $\mathrm{He}^{4}$, Deuterium and $\mathrm{Li}^{7}$ abundances which determine $\delta N_{\nu}$.

Clearly to satisfy the inflationary constraint of $\Omega_{T O T}=1$, we need $\Omega_{\Lambda} \simeq 0.7$. These kinds of numbers for cold dark matter density apparently emerge from current type I supernovae observations. It is interesting to note that if one were to require that $\Omega_{C D M}=1$, the mirror model would require that $\zeta$ be much larger (more than 100) which would then create difficulties in understanding both the neutrino data and the microlensing anomalies. Thus mirror baryons seem to have just the right properties to be the cold dark matter of the universe.

\section{EXPLAINING THE MICROLENSING ANOMALY}

Let us now turn to show how the mirror model accounts for the microlensing observations. We start with the four equations of stellar structure:

$$
\begin{gathered}
d P / d r=-G \rho(r) M(r) / r^{2} \\
d M(r) / d r=4 \pi r^{2} \rho(r) \\
L(r) / 4 \pi r^{2}=-(16 / 3) \sigma_{S B}\left(T^{3} / \rho \kappa\right) d T / d r \\
d L / d r=4 \pi r^{2} \epsilon(r) \rho(r)
\end{gathered}
$$

where $\kappa(r)$ is the opacity (cross section per unit mass) at radius $r, \sigma_{S B}$ the Stefan-Boltzmann constant, $\mathrm{L}(\mathrm{r})$ the luminosity at radius $r$, and $\epsilon(r)$, the rate of energy generation per unit mass at radius $r$. We will need three terms in the equation of state (below) taken one or two at a time:

$$
P=(\rho / m) k T+\left(4 \sigma_{S B} / 3 c\right) T^{4}+\left(h^{2} / 2 m_{e}\right)(3 / 8 \pi)^{2}(\rho / m)^{5 / 3}
$$

where the three terms represent gas pressure, radiation pressure, and (non-relativistic) degenerate electron pressure. $m$ is the nucleon mass, $m_{e}$ that of the electron. We have neglected such niceties as keeping track of how many objects there are for each $m$ of gas $(2$ for $\mathrm{H}, 3 / 4$ for $\mathrm{He}$, etc) 
We will make standard, illuminating if crude, approximations 19 in order to understand the $\zeta$ behavior of the solutions to the above equations. First we write

$$
P=\rho G M / R, \quad \rho=3 M / 4 \pi R^{3}
$$

where $P$ and $\rho$ are roughly core averages. Here $M$ and $R$ are mass and radius of the star. This gives the useful relation

$$
P=(4 \pi / 3)^{1 / 3} G M^{2 / 3} \rho^{4 / 3}
$$

To find the maximum mass of a (main sequence) mirror star, which is of interest to us, we note that as the mass of the star gets bigger, the core temperature rises. Therefore, of the three terms in the expression for the pressure in the Equation 8, we expect $P_{g}$ and $P_{r}$ to dominate. Following Phillips [19], we parameterize them as fractions of the total pressure $P$ as below:

$$
P_{g}=\beta P, \quad P_{r}=(1-\beta) P
$$

We eliminate $T$ and solve for $\mathrm{P}$ from this parameterization to obtain

$$
\beta P=\left[(\rho k / m)^{4}\left(\beta^{-1}-1\right) /\left(4 \sigma_{S B} / 3 c\right)\right]^{1 / 3}
$$

Using Equation (10) again then gives

$$
M_{\max } \sim\left[(1-\beta) c / \sigma_{S B}\right]^{1 / 2} G^{-3 / 2}(k / m)^{2} / \beta^{2}
$$

As $\beta$ approaches 1 , the energy density is increasingly dominated by photons (relativistic particles) and stars become unstable. Taking a cutoff around $\beta \sim 1 / 2$ gives a maximum stellar mass around $70 M_{\odot}$. Thus the range for stars is roughly $0.07 M_{\odot}$ to $70 M_{\odot}$. From Equation (13) one sees, in the approximation that instability sets in at the same $\beta$ independent of $\zeta$, that $M_{\max }$ varies as $\zeta^{-2}$. For $\zeta=15$, we get for the maximum mass of the mirror star $0.5 M_{\odot}$, which is of the same order of mass as the MACHO microlensing events. Our model therefore provides a resolution of the microlensing anomaly that avoids the strong constraints of Freese et al [20] for familiar sector white dwarfs.

We want to point out here that we do not expect all of mirror dark matter to condense to form mirror stars. Instead, we would expect it to be in the form of a mixture of mirror dust and mirror stars. In this connection, it has been noted recently 21] that current upper limits to scattering optical depths for Thomson scattering in early universe suggests that compact objects of any kind cannot be the main dark matter constituent. This would also suggest a mixed picture of the kind mentioned.

\section{SELF INTERACTION OF MIRROR MATTER AND HALO CORE DENSITY PROBLEM}

As noted in the introduction, there appear to be indications from the core density profile of dwarf and low surface brightness galaxies [5,6] that the dark matter may need to be endowed with a significant self interaction. According to the analysis of Spergel and Stenhardt 
[8], the self interaction must be such that the collision cross section of dark matter particles should be of order $10^{-23}(\mathrm{~m} / \mathrm{GeV}) \mathrm{cm}^{2}$ for a one $\mathrm{GeV}$ particle corresponding to a DM mean free path of order of $\lambda_{s s}=300 \mathrm{kpc}$ in a gas with number density given by $\rho_{D M} / \mathrm{m}$. Also we note that the cross section would scale linearly with the mass of the dark matter particle. The mean free path requirement cannot be met by the neutralino or the axion but is met quite naturally by the mirror dark matter forces [22].

Two obvious kinds of self interactions are the self interactions due to inter-atomic forces and nuclear forces. The latter are not very effective as can be seen by the following crude argument. From pure $\zeta$ scaling, we can infer that $\sigma_{N^{\prime} N^{\prime}} \approx \sigma_{N N} \zeta^{-2}$. The value of $\zeta=20$ would put the mirror nucleon cross section to be of order $10^{-26} \mathrm{~cm}^{2}$, which is much too small. Note that for $\zeta=20$, one would need a cross section of order $10^{-22} \mathrm{~cm}^{2}$ to get $\lambda=\lambda_{s s}$. On the other hand, one would expect that there can be scattering of the dark matter particles (mirror atoms) due to inter-atomic forces. A crude estimate of such cross sections is given by $\sigma_{H^{\prime} H^{\prime}} \approx \pi\left(a_{0}^{\prime}\right)^{2}$, where $a_{0}^{\prime}$ is the Bohr radius of the mirror hydrogen atom. One can then estimate the mean free path of the dark matter particles in the mirror model to be $\lambda_{D M} \approx 3 \pi \zeta^{3} \times 10^{16} \mathrm{~cm}$. For $\zeta=20$, this gives $\lambda_{D M} \approx 0.3 \mathrm{kpc}$, which is not far below the required values for explaining the halo core density profile. Note that Spergel and Steinhardt [8] require $\lambda_{D M} \approx \mathrm{kpc}$ to mpc. On the other hand, one could take the value of $\zeta=30$ and thereby get $\lambda \approx$ one kpc.

We thus feel that mirror dark matter presents the best scenario for understanding the halo core density profile using a self interacting dark matter model. Of course more detailed numerical work is needed to confirm these qualitative conclusions.

\section{STRUCTURE FORMATION}

Finally, we use the remainder of this article to make plausible that, in spite of the $\zeta^{2}$ decrease in cross sections for most processes, the facts that (a) structure formation begins earlier for the mirror sector (because recombination occurs before matter-radiation equality) and (b) the higher mirror temperatures for the same processes, than familiar temperatures, permit formation of galactic and smaller structures. In doing this, we will make use of our previous work in [23] and [16], as well as that of Tegmark et al [24].

Much of the work of [23] can be carried over to the present work, after suitable modification to take into account the fact that, in the current model, the proton mass scales as $\zeta$. Here, we will assume that primordial perturbations are "curvature" or "adiabatic" perturbations. This means that the scale of the largest structures are set by mirror Silk damping [25]. $\gamma^{\prime}$ diffusion wipes out inhomogeneities until the $\gamma^{\prime}$ mean free path,

$$
\lambda^{\prime}=\left[\sigma_{T} \zeta^{-2} n_{e}^{\prime}\right]^{-1}
$$

where $\zeta^{-2} \sigma_{T}$ is the mirror matter Thomson cross section and $n_{e}^{\prime}$ is its electron number density, becomes greater than one third the horizon distance $(c t)$. Silk damping turns off because the $\lambda^{\prime}$ increases as $z^{-3}$ while $c t$ only increases as $z^{-2}$.

First, we compute, from Silk damping, the masses of the largest structures in this picture. Structure formation starts with mirror sector particles, and familiar sector particles later fall into these. For numerical values below, we will take, $h=0.7$ and $\Omega_{B^{\prime}}=0.2$. We pick 
$t \sim\left(z_{1} / z\right)^{2} s$ with $z_{1}=4 \times 10^{9}$ and $n_{B^{\prime}}=\Omega_{B^{\prime}} \rho_{c} z^{3} /\left(\zeta m_{p}\right)$ with $\rho_{c}=1.9 h^{2} \times 10^{-29} \mathrm{~g} / \mathrm{cm}^{3}$. Silk damping stops at around $z_{s d} \sim 8 \zeta^{3}$ which gives

$$
\begin{aligned}
\lambda_{s d} \sim 2.5 & \times 10^{27} / \zeta^{6} \mathrm{~cm} \\
M_{s d} & \sim 10^{54} / \zeta^{9} \mathrm{gm}
\end{aligned}
$$

Note that, for $\zeta \sim 10$, this is about the mass (and size) of a large galaxy. This coincidence could be an important factor in understanding galaxy sizes should this model correspond to reality.

As in [23], we parametrize the separation of $M_{s d}$ from the expanding universe as taking place at

$$
z_{s t o p}=z_{M} z_{s d}
$$

with

$$
R_{G}=\lambda_{s d} / z_{M}
$$

After violent relaxation we have for the proton temperature

$$
T_{p}=G M_{G} \zeta m_{p} / R_{G} \sim 10^{-4} z_{M} / \zeta^{2} \text { ergs }
$$

with $\rho$, outside the central plateau, given by

$$
\rho(R)=A / R^{2} \sim 10^{26} z_{M} /\left(\zeta^{3} R^{2}\right) \mathrm{gm} / \mathrm{cm}^{3}
$$

We now turn to the question of whether this isothermal sphere is likely to fragment and form mirror stars. For this we compute the amount of mirror molecular hydrogen since it is its collisional excitation (and subsequent radiation) that is believed to be the chief mechanism that provides cooling for formation of stars. If the rate for this mechanism is faster than the rate for free fall into the mass of the structure at issue, we can expect local regions to cool fast enough to result in fragmentation of that structure. We do here a very rough estimate of mirror galaxy fragmentation into mirror globular clusters, using the results of [24], but leave to a more detailed work further fragmentation into the $0.5 M_{\odot}$ structures predicted in [16].

Reference [24] give a useful approximation to their numerical results for the fraction of molecular hydrogen, $f_{2}\left(f_{0}\right.$ denotes its primordial value):

$$
f_{2}(t)=f_{0}+\left(k_{m} / k_{1}\right) \ln \left[1+x_{0} n k_{1} t\right)
$$

where, as a first try, $k_{m}$ can be taken as just the rate for $H+e^{-} \rightarrow H^{-}+\gamma$ (which they conveniently give as about $2 \times 10^{-18} \mathrm{~T}^{.88} \mathrm{~cm}^{3} \mathrm{~s}^{-1}$ ), while $k_{1}$ is the rate for $\mathrm{H}^{+}+e^{-} \rightarrow H+\gamma$ $\left(2 \times 10^{-10} T^{-0.64} \mathrm{~cm}^{3} \mathrm{~s}^{-1}\right)$. Equation (8) is the result of $\mathrm{H}_{2}$ production from the catalytic reactions $H+e^{-} \rightarrow H^{-}+\gamma$ followed by $H+H^{-} \rightarrow H_{2}+e^{-}$competing against the recombination reaction that destroys the catalyst, free electrons, (approximately) as $1 / t$ (assuming constant density). Our goal here is to show from Equation (20) that it is plausible that $f_{2}$, the fraction of molecular hydrogen, rises from its primordial value of $10^{-6}$ to the region above $10^{-4}$ where cooling tends to be competitive with free fall. 
First, we note that, if $k=<v \sigma>\sim A T^{\gamma} \mathrm{cm}^{3} / s$, for familiar e and $\mathrm{p}$, we expect that, for mirror e and p, scaling with $\zeta$ to go as $\zeta^{-(2+\gamma)} A T^{\gamma}$, since $\sigma$ must go as $\zeta^{-2}$, all factors of $T$ must be divided by some combination of $m_{e}$ and $M_{p}$, both of which go as $\zeta$ (making this model much easier to compute from than that of [23]).

We now estimate fragmentation. From Equation (18) we see that the galactic temperature should begin at about $10 \mathrm{eV}$ at a time when the cosmic temperature is about $1 \mathrm{eV}$ and the cosmic gamma number density is about $10^{9} / \mathrm{cm}^{3}$. The rate for "compton cooling" is very fast at this high density (unlike at later times for the familiar case) and there should be rapid cooling to about $1 \mathrm{eV}$. We can now compute the Jeans length for fragments as a function of distance $\mathrm{R}$ from galaxy central. We use

$$
\rho_{J}=(T / G m)^{3} / M^{2}
$$

If we set the Jeans mass, $M$, to $4 \pi r^{3} \rho_{J} / 3$, we can solve for $r$ obtaining (if we are careful to convert $T$ in Equation (6) from ergs)

$$
r=R\left[10^{-7} \zeta^{2} / z_{M}\right]^{1 / 2} \sim 10^{-2} R
$$

Now inserting into Equation (20) gives the coefficient of the log term on the order of $10^{-3.5}$ and the coefficient of $t$ in the argument varying from $10^{-13}$ to $10^{-17}$ as $R$ varies from

1 to 100 kiloparsecs while the free fall time $\left((G \rho)^{-1 / 2}\right)$ varies from $10^{14}$ to $10^{16}$. This would appear to indicate the likelihood of fragmentation of the original Silk damping structure into smaller units, and the eventual formation of the $0.5 M_{\text {odot }}$ black holes that explain the microlensing events of [16].

\section{CONCLUSION}

We have argued that the asymmetric mirror model [13], originally proposed to solve neutrino puzzles and subsequently advocated [16,17] as providing an alternative dark matter candidate has the advantage of resolving the microlensing anomaly and possibly the core density problem of dark halos. Possible tests of these models are to narrow the allowed values of $\delta N_{\nu}$ from more accurate observation of deuterium, $\mathrm{He}^{4}$ and $\mathrm{Li}^{7}$ and observing whether further accumulation of MACHO candidates lie in the mass between 0.1- 1 solar mass. Needless to say that if the underground searches for the cold dark matter now under way lead to a positive signal, mirror matter cannot be the dominant component of the dark matter of the universe.

The work of R. N. M. is supported by the National Science Foundation grant under no. PHY-9802551 and the work of V. L. T. is supported by the DOE under grant no. DE-FG03-95ER40908. 


\section{REFERENCES}

[1] For a survey of recent observational and theoretical advances in this area see, Dark Matter in Astro and Particle Physics ed. by H. V. Klapdor-Kleingrothaus and Y. Ramachers (World Scientific), 1996 and H. V. Klapdor-Kleingrothaus and L. Baudis, (IOP Publication), 1998.

[2] C. Alcock et al. Ap. J. 486, 697 (1997); R. Ansari et al. A \& A 314, 94 (1996).

[3] E. Gates, G. Gyuk and M. Turner, Phys. Rev. D 53, 4138 (1996).

[4] R. Flores and J. Primack, Ap. J. 427, L1 (1994).

[5] B. Moore, Nature, 370, 629 (1994); B. Moore et al, astro-ph/9907411.

[6] W. J. G. de Blok and S. S. McGough, MNRAS, 290, 533 (1997).

[7] J. F. Navarro, C. S. Frenk and S. White, Ap. J. 462, 563 (1996).

[8] D. Spergel and P. Steinhardt, astro-ph/9909386.

[9] S. Hannestad, astro-ph/9912558; S. Hannestad and R. Sherrer, astro-ph/0003046.

[10] Craig J. Hogan and J. J. Dalcanton, astro-ph/0002330.

[11] E. Carlson, M. Machacek and L. J. Hall, Ap. J. 398, 43 (1992); A. A. de Laix, R. K. Shaeffer and R. J. Sherrer, Ap. J. 452, 495 (1995).

[12] T. D. Lee and C. N. Yang, Phys. Rev. 104, 254 (1956); K. Nishijima, private communication; Y. Kobzarev, L. Okun and I. Ya Pomeranchuk, Yad. Fiz. 3, 1154 (1966); M. Pavsic, Int. J. T. P. 9, 229 (1974); S. I. Blinnikov and M. Y. Khlopov, Astro. Zh. 60, 632 (1983); E. W. Kolb, D. Seckel and M. Turner, Nature, 514, 415 (1985); R. Foot, H. Lew and R. Volkas, Phys. Lett. B 272, 67 (1991).

[13] Z. Berezhiani and R. N. Mohapatra, Phys. Rev. D 52, 6607 (1995); Z. Berezhiani, A. Dolgov and R. N. Mohapatra, Phys. Lett. B 375, 26 (1996).

[14] R. Foot and R. Volkas, Phys. Rev. D 52, 6595 (1995).

[15] For other papers on the subject, see H. Hodges, Phys. Rev. d 47, 456 (1993); M. Collie and R. Foot, Phys. Lett. B432, 134 (1998); B. Brahmachari and R. N. Mohapatra, hepph/9805429; Z. Silagadze, hep-ph/9908208; N. F. Bell and R. Volkas, Phys. Rev. D 59, 107301 (1999); N. Bell, hep-ph/0003072; R. Foot and S. N. Ginnenko, hep-ph/0003278; for a recent review, see Z. Silagadze, hep-ph/0002255.

[16] R. N. Mohapatra and V. L. Teplitz, Phys. Lett. B 462, 302 (1999).

[17] S. I. Blinnikov, astro-ph/9801015; Z. Berezhiani, hep-ph/9602326.

[18] Z. Berezhiani et al. ref. [13]; V. Berezinsky and A. Vilenkin, hep-ph/9908257.

[19] D. Clayton, Principle of Stellar Evolution and Nucleosynthesis, McGraw-Hill, New York (1968); A. C. Phillips, Physics of Stars, John-Wiley (1996); W. K. Rose, Advanced Stellar Astrophysics, Cambridge University Press (1998).

[20] D. S. Graff and K. Freese, Ap. J. 456, L49 (1996); D. S. Graff, G. Laughlin and K. Freese, Ap. J. 499, 7 (1998); For a review, see K. Freese, B. Fields and D. Graff, astro-ph/9901178.

[21] M. C. Miller, astro-ph/0003176.

[22] R. N. Mohapatra and V. L. Teplitz, astro-ph/0001362.

[23] R. N. Mohapatra and V. L. Teplitz, Ap. J. 478, 29 (1997).

[24] M. Tegmark et al. Ap. J. 474, 1, (1997).

[25] J. Silk, Ap. J. 211, 638 (1977). 